\title{
Consultations virtuelles ou en personne en soins primaires
}

\author{
Logan Sept Jessica Kirkwood MD CCFP(AM) Christina S. Korownyk MD CCFP
}

\section{Question clinique}

Quelle est l'exactitude diagnostique lors des consultations virtuelles par rapport aux consultations en personne pour des présentations indifférenciées?

\section{Résultats}

Selon des données probantes limitées et de faible niveau, l'exactitude diagnostique lors de consultations virtuelles se situait entre 71 et $91 \%$, comme évaluée en se fondant sur des patients standardisés ou des revues de cas à 3 mois. L'exactitude ou la concordance du diagnostic dans les soins virtuels semble semblable à celle des consultations en personne. Ces études ne se penchent pas sur la continuité des soins ni sur les résultats chez les patients.

\section{Données probantes}

- Dans une étude sur le diagnostic auprès d'une cohorte de 97 adultes à leur première visite dans une clinique de médecine générale, les consultations en personne étaient suivies par une vidéoconférence avec un médecin différent ${ }^{1}$.

-L'exactitude diagnostique n'était pas significativement différente entre les consultations en personne (83\%) et les vidéoconférences ( $80 \%$ ). Les présentations les plus courantes étaient d'ordre respiratoire (22\%), digestif (19\%) ou circulatoire (10\%); $57 \%$ des présentations étaient de nature aiguë et $43 \%$ de nature chronique.

-Limites: tous les patients avaient d'abord été évalués en personne, et il n'y avait pas de suivi à long terme.

- Dans un audit de 599 consultations virtuelles avec 67 patients standardisés présentant 1 de 6 problèmes (douleur à la cheville, pharyngite virale ou bactérienne, infection récurrente des voies urinaires, rhinosinusite et lombalgie) $)^{2}$ l'exactitude diagnostique variait selon la présentation ( $71 \%$ pour la rhinosinusite, $91 \%$ pour les infections des voies urinaires).

-Il n'y avait pas de différence dans l'exactitude diagnostique entre les vidéos ou par téléphone.

-Limites: problèmes limités, uniques; patients non réels.

- Dans une étude transversale en soins primaires, on a choisi aléatoirement 175 adultes qui auraient 1 consultation en vidéoconférence et 1 en personne, ou encore 2 consultations en personne. Les 2 consultations étaient avec des médecins différents ${ }^{3}$. La concordance diagnostique n'était pas significativement différente entre les groupes (84 C. $80 \%$ ).
-Limites:petit nombre de sujets; l'étude portait à la fois sur des problèmes indifférenciés et sur des maladies chroniques.

- Des revues systématiques sur les soins virtuels ont publié des données sur l'accès, la satisfaction, les coûts et la charge clinique; par ailleurs, les données probantes sur l'exactitude diagnostique sont limitées ${ }^{4,5}$.

\section{Contexte}

- Parmi les préoccupations entourant les consultations virtuelles figurent la difficulté d'établir des rapports, et les risques posés au suivi et à la continuité des soins ${ }^{6,7}$.

-La continuité des soins se traduit à long terme par une baisse dans les coûts, les hospitalisations et la mortalité 8,9 .

- Il est difficile d'évaluer les erreurs diagnostiques. Des études observationnelles ${ }^{10}$ dont la période de suivi était plus longue estimaient que le taux d'erreurs diagnostiques chez des patients en consultation externe se situait à environ $5 \%$.

- La plupart des diagnostics "non reconnus" étaient des problèmes courants en soins primaires: la pneumonie $(6,7 \%)$, l'insuffisance cardiaque $(5,7 \%)$, l'insuffisance rénale aiguë $(5,3 \%)$ et le cancer $(5,3 \%)^{11}$.

\section{Mise en pratique}

De nouvelles lignes directrices sur la mise en œuvre pratique des soins virtuels font lentement leur apparition. L'Association médicale canadienne a élaboré un répertoire d'idées et de suggestions pratiques pour intégrer les consultations virtuelles dans la pratique au quotidien ${ }^{12}$. Étant donné que la continuité des soins est associée à de meilleurs résultats, les soins virtuels qui facilitent la continuité devraient être privilégiés, plutôt que les consultations virtuelles de cliniciens avec qui les patients n'ont pas de relations établies.

M. Sept est étudiant en médecine à l'Université de l'Alberta à Edmonton. La Dre Kirkwood est médecin de famille au Boyle McCauley Health Centre à Edmonton (Alberta). La

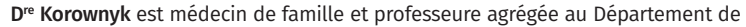
médecine familiale de l'Université de l'Alberta.

Intérêts concurrents

Aucun déclaré

Les opinions exprimées dans Outils pour la pratique sont celles des auteurs et ne reflètent pas nécessairement le point de vue et les politiques du Collège des médecins de famille de l'Alberta.

Références

1. Ohta M, Ohira Y, Uehara T, Keira K, Noda K, Hirukawa M et coll. How accurate are first visit diagnoses using synchronous video visits with physicians? Telemed J E Health 2017;23(2):119-29.

2. Schoenfeld AJ, Davies JM, Marafino BJ, Dean M, DeJong C, Bardach NS et coll. Variation in quality of urgent health care provided during commercial virtual visits. JAMA Intern Med 2016;176(5):635-42.

3. Dixon RF, Stahl JE. A randomized trial of virtual visits in a general medicine practice. J Telemed Telecare 2009;15(3):115-7. 
4. Flodgren G, Rachas A, Farmer AJ, Inzitari M, Shepperd S. Interactive telemedicine: effects on professional practice and health care outcomes. Cochrane Database Syst Rev 2015;(9):CD002098.

5. Lake R, Georgiou A, Li J, Li L, Byrne M, Robinson M et coll. The quality, safety and governance of telephone triage and advice services-an overview of evidence from systematic reviews. BMC Health Serv Res 2017;17(1):614.

6. Hammersley $\mathrm{V}$, Donaghy $\mathrm{E}$, Parker $\mathrm{R}, \mathrm{McNeilly} \mathrm{H}$, Atherton $\mathrm{H}$, Bikker A et coll. Comparing the content and quality of video, telephone, and face-to-face consultations: a non-randomised, quasi-experimental, exploratory study in UK primary care. $\mathrm{Br}$ J Gen Pract 2019;69(686):e595-604.

7. Hardcastle L, Ogbogu U. Virtual care: enhancing access or harming care? Healthc Manage Forum 2020;33(6):288-92.

8. Bazemore A, Petterson S, Peterson LE, Bruno R, Chung Y, Phillips RL Jr. Higher primary care physician continuity is associated with lower costs and hospitalizations. Ann Fam Med 2018;16(6):492-7.

9. Pereira Gray DJ, Sidaway-Lee K, White E, Thorne A, Evans PH. Continuity of care with doctors-a matter of life and death? A systematic review of continuity of care and mortality. BMJ Open 2018;8(6):e021161.

10. Singh $\mathrm{H}$, Meyer AND, Thomas EJ. The frequency of diagnostic errors in outpatient care: estimations from three large observational studies involving US adult populations. BMJ Qual Saf 2014;23(9):727-31.
11. Singh $\mathrm{H}$, Giardina TD, Meyer AND, Forjuoh SN, Reis MD, Thomas EJ. Types and origins of diagnostic errors in primary care settings. JAMA Intern Med 2013;173(6):418-25.

12. Dermer M. Virtual care playbook. Ottawa, ON: CMA; 2020. Accessible à : https://www.cma ca/sites/default/files/pdf/Virtual-Care-Playbook_mar2020_E.pdf. Réf du 1e oct. 2020.

Cet article donne droit à des crédits d'autoapprentissage certifiés Mainpro+. Pour obtenir des crédits, rendez-vous à www.cfp.ca et cliquez sur le lien Mainpro+.

Can Fam Physician 2020;66:905-6. DOI: 10.46747/cfp.6612905

This article is also in English on page 904.

Les articles d'Outils pour la pratique dans Le Médecin de famille canadien (MFC) sont une adaptation d'articles publiés dans le site web du Collège des médecins de famille de l'Alberta (CMFA) qui résument les données médicales probantes en insistant sur des questions particulières et des renseignements susceptibles de modifier la pratique. Les résumés du CMFA et la série dans le MFC sont coordonnés par $\mathbf{D}^{r} \mathbf{G}$. Michael Allan, et les résumés sont rédigés conjointement par au moins 1 médecin de famille en pratique active et ils font l'objet d'une révision par des pairs. Vous êtes invités à faire part de vos commentaires à toolsforpractice@cfpc.ca. Les articles archivés sont accessibles sur le site web du CMFA: www.acfp.ca. 